\title{
Resultados e interpretación
}

Teniendo en cuenta el objetivo de la investigación, a continualos aportes que tanto los documentos de la Iglesia y de la Orden de Predicadores como los cuestionarios aplicados aportan en la construcción de una propuesta curricular para la formación de los prenovicios dominicos en Colombia.

\section{Desde el análisis documental}

De acuerdo con la aplicación del instrumento llamado Resumen analítico de investigación (RAI), en 23 documentos se obtuvieron las siguientes categorías, sintetizadas y relacionadas con los componentes del currículo, a saber: fines de la formación, contenidos, etapas, responsables y tiempo.

\section{Magisterio de la Iglesia}

- Fines de la formación: formar hombres capaces de dar testimonio del seguimiento de Jesucristo a través de la consagración religiosa y del ministerio sacerdotal. 
- Contenidos: dimensión espiritual, intelectual, pastoral y disciplinar.

- Etapas: prenoviciado, noviciado, votos temporales.

- Responsables: formandos, maestros y superiores.

- Tiempo: mínimo seis meses, máximo tres años.

\section{Magisterio de la Orden de Predicadores}

- Fines de la formación: formar personas fieles al seguimiento de Jesucristo a través del modo ideado por santo Domingo de Guzmán.

- Contenidos: desarrollo de la dimensión humana, religiosa, intelectual y pastoral.

- Etapas: aspirantado, prenoviciado, noviciado, estudiantado (filosofía y teología), formación permanente.

- Responsables: formando.

- Tiempo: un año.

Respecto de los lineamientos de la Orden de Predicadores y teniendo en cuenta el énfasis de la investigación en la formación de los frailes, de manera especial, en su primera etapa de formación institucional, a continuación se rescatan cinco lineamientos.

La Orden de Predicadores ha planteado cuatro ejes para la formación inicial de los frailes: el humano, el religioso, el intelectual y el pastoral. Estos ejes constituyen el fundamento para la formación integral de la persona, en el que el joven prenovicio comienza a conocer un proyecto de vida que le permite optar libre y sin coacción alguna para continuar o no en su proceso de formación para la vida religiosa.

Adicionalmente, dichos ejes de la formación están directamente ligados con la espiritualidad o carisma de la Orden de Predicadores. 
Por otra parte, se concibe que la formación de la persona dura toda la vida, y el primer responsable es el mismo formando, incluso después de culminada su formación institucional.

Por último, la tarea o el oficio del formador es acompañar, guiar y orientar, por medio del diálogo y la confianza, a los jóvenes que desean optar por el seguimiento de Cristo a través del carisma y la espiritualidad de la Orden de santo Domingo de Guzmán.

\section{Fines de la formación en otras comunidades religiosas masculinas}

- Fines de la formación: formar personas con una madurez humana, espiritual, fraterna, afectiva y evangélica.

- Contenidos: desarrollo humano, intelectual, espiritual, (carisma propio de la comunidad) y misionero.

- Etapas: postulantado (prenoviciado), noviciado, filosofado y teologado.

- Responsables: formandos, formadores y superiores.

- Tiempo: de seis meses a dos años.

\section{Desde los cuestionarios aplicados ${ }^{1}$}

Retomando la muestra inicial calculada en 33 formandos, distribuidos por cada año, respondieron el cuestionario 30, es decir, el 90.9\%, distribuidos de la siguiente manera: año 2011, 9 de 11 formandos, equivalente al $81.8 \%$; año 2012, 9 de 10 formandos, equivalente al $90 \%$ y, año 2013, 12 de 12 formandos, equivalente al 100\% de los encuestados.

1 Cfr. <https://docs.google.com/forms/d/1lQ2PUC0c2duPSCsnlgvdROlS55Jp8 cbjZPnWDUfjh9g/viewanalytics $>$. 
Tabla 7. Estadística general de formandos encuestados

\begin{tabular}{|c|c|l|c|r|}
\hline Año & Formandos & \multicolumn{1}{|c|}{ Etapa de formación } & Respuesta & \multicolumn{1}{c|}{$\%$} \\
\hline 2011 & 11 & Primer año de Filosofía - Bogotá & 9 & $81.8 \%$ \\
\hline 2012 & 10 & Noviciado - Chiquinquirá & 9 & $90.0 \%$ \\
\hline 2013 & 12 & Prenoviciado - Tunja & 12 & $100.0 \%$ \\
\hline Total & 33 & & 30 & $90.9 \%$ \\
\hline
\end{tabular}

Fuente: elaboración del autor.

Los resultados se presentan bajo dos criterios: ejes de la formación dominicana y elementos del currículo, elementos necesarios para una propuesta curricular de un plan de formación en el prenoviciado que, según los criterios de la Orden de Predicadores, recibe el nombre de Ratio Formationis Prenoviciado (RFPR)

\section{Características generales de los formandos que atendieron a la encuesta planteada vía internet ${ }^{2}$}

\section{Edad}

El $40 \%$ de los formandos tiene una edad que oscila entre 16 y 20 años. El $37 \%$ de los formandos tiene una edad que oscila entre 20 y 25 años. El 7\% de los formandos tiene una edad que oscila entre 30 y 35 años.

La interpretación de estos datos nos permite concluir que la población actual es muy joven, prácticamente el $77 \%$ de los formandos son menores de 25 años. Si se suman los demás años de formación en el estudiantado (Filosofía y Teología), se puede deducir que un formando culminará sus estudios institucionales antes de los 28 años, lo cual genera expectativas frente a los retos que constituyen el proyecto comunitario de la Provincia Dominicana de Colombia para los próximos años.

2 Cfr. <https://docs.google.com/forms/d/1lQ2PUC0c2duPSCsnlgvdRO1S55Jp8cbjZPnWDUfjh9g/edit>. 


\section{Profesiones}

El grupo de formandos lo conforman seis personas con estudios de pregrado y dos técnicos, distribuidos así: dos abogados, un contador público, un ingeniero electrónico, un odontólogo, un licenciado en filosofía, un tecnólogo en comunicación ilustración y un técnico en administración de sistemas.

\section{Ejes transversales de la formación dominicana}

Tomando como base los cuatro elementos transversales que deben estar en las etapas de la formación institucional en la $\mathrm{Orden}^{3}$, en la ilustración 1 se presentó el paralelo entre los lineamientos del Magisterio de la Iglesia y de la Orden de Predicadores para la formación de los sacerdotes y consagrados, los cuales se constituyen en los pilares del carisma dominicano, según se trata en las siguientes secciones.

\section{Vida comunitaria}

Desde el humanismo dominicano-tomista, la formación integral centra su atención en la persona. Los formandos consideran que la promoción y el desarrollo humano, además de la creación y estructuración de un proyecto de vida, son los principales elementos que permiten la construcción de lazos de fraternidad entre sus nuevos hermanos. Vale la pena recordar aquí la Regla de San Agustín ${ }^{4}$, sobre la cual se ancla la vida dominicana, en concordancia con el texto de la Sagrada Escritura: "Todas las cosas les eran comunes y se distribuía a cada uno según su necesidad" ${ }^{\text {. }}$.

3 Byrne, Damian. Carta sobre la formación en todas sus etapas. Roma, Curia Generalicia, 1991.

4 San Agustín. La régle de Saint Augustin I. La tradición manuscrite. París. 1969, pp. 417-437.

5 Biblia de Jerusalén. Hechos de los Apóstoles 4,32-35. Bilbao, Desclée de Brouwer, 2009. 


\section{Vida espiritual}

La oración cristiana mantiene vivo, activo y dinámico el espíritu de diálogo con Dios y con los hermanos. Los formandos consideran que el seguimiento de Cristo, la Eucaristía y la experiencia de fe son los elementos fundamentales sobre los que se construye el sentido de su vida espiritual, lo cual les permite avanzar en el conocimiento de los modelos de vida religiosa.

La oración, el ayuno y la mortificación, según las Constituciones de la Orden de Predicadores ${ }^{6}$, se entienden como la vida regular de los frailes en donde se alaba, bendice y celebra la presencia de Dios en la vida del ser humano, a través de salmos, himnos, la lectura y meditación de la Palabra de Dios, conocida como la Lectio Divina.

\section{Vida académica-intelectual}

Santo Domingo insertó profundamente en el ideal de su Orden el estudio dirigido al ministerio de la salvación, llevaba siempre consigo el Evangelio de San Mateo y las epístolas de San Pablo, condujo y animó a los frailes a las escuelas y les envió a las grandes ciudades "para que estudiaran, predicaran y fundaran Convento"7.

El estudio en la Orden de Predicadores se constituye en uno de los ejes transversales durante la formación institucional y permanente, puesto que a través de este se prepara al predicador para el servicio doctrinal de la Iglesia y de toda la humanidad. Esto responde a que se considera que el crecimiento intelectual de los formandos y el subsecuente desarrollo de sus habilidades cognitivas les permiten avanzar en su proceso durante el prenoviciado.

Para el $17 \%$ de los encuestados, la estructura académica les permitió elaborar y desarrollar un método de estudio, construir conocimiento y cualificarse como persona.

6 Orden de Predicadores. Libro de constituciones y ordenaciones (LCO). Roma, Curia Generalicia, 2010, n. ${ }^{\circ} 10-18$.

7 Ibíd., n. ${ }^{\circ} 76-77$. 


\section{Vida apostólica}

La predicación no es solo una función, una tarea, una misión. Es una forma de vida, un estilo de vida, es la vita vere apostolica que Domingo quiso para sí y para sus seguidores ${ }^{8}$. Domingo concibió su proyecto fundacional en función de la predicación, la cual acontece en el anuncio teológico del Evangelio, comunicando la gracia y la esperanza cristiana, como esencia del mensaje de Jesús de Nazareth para los necesitados y desfavorecidos de nuestro mundo.

Este anuncio se logra a través del desarrollo de las actividades apostólicas, con las cuales se pretende que los formandos conozcan e incursionen en la vida de predicación en la Orden, en contacto con las realidades eclesiales de la ciudad de Tunja.

Principalmente, para un formando esta experiencia le generó sensibilidad y solidaridad, el $15 \%$ de los encuestados así lo afirma. De igual manera, suscitó un sentido de compromiso, liderazgo y creatividad; propios del interés por la Misión de la Orden de Predicadores.

\section{Elementos del currículo}

Dentro del diseño e implementación de la encuesta, se plantearon los elementos curriculares que, según los hallazgos teóricos, plasmados en la ilustración 7, orientan y guían los procesos de enseñanza y aprendizaje.

\section{Propósitos educativos}

Para la Orden de Predicadores, solo hay un signo de identidad, un "código genético" para los miembros de la Orden, de la familia dominicana: es la predicación para la salvación de la humanidad ${ }^{9}$, el ministerio de la Palabra, la misión evangelizadora.

En sintonía con la misión de la Orden, el objetivo del prenoviciado es preparar al aspirante al noviciado, principalmente con una instrucción catequética y cierta experiencia de vida comunitaria, así como

8 Orden de Predicadores, Actas del Capítulo General Electivo. Roma, 2010, p. 38 .

9 Orden de Predicadores, Constitución Fundamental LCO 1 n. ${ }^{\circ}$ IV, Roma, 2010. 
ofrecer a la Orden la oportunidad de discernir acerca de la idoneidad del aspirante para abrazar la vida dominicana.

Ante este propósito de la formación en el prenoviciado, el 100\% de los encuestados consideraron que este se cumplió a cabalidad, y de manera especial piensan que la responsabilidad y la autonomía, así como la madurez humana, son los factores más destacados en cada uno de los procesos de acompañamiento. Así lo señaló uno de los formandos: "Porque la formación se centra en la persona como parte de una comunidad. En la etapa del prenoviciado, el acompañamiento para el discernimiento ha sido oportuno y adecuado para poder optar con responsabilidad por la vida religiosa". De igual manera, otro de los formandos expresa las siguientes palabras: "El sentir que he crecido integralmente en las dimensiones, humano-comunitaria, intelectual, espiritual, pastoral, social, afectiva, etc., me hace pensar que es efectivo y fructífero este proceso de acompañamiento".

\section{Los contenidos de la formación}

Frente a los contenidos que se enmarcan en cada etapa del proceso de formación en el prenoviciado, los formandos consideran que desarrollaron principalmente la dimensión humana y cristiana (100\%), comunitaria (96\%), intelectual (95\%). La dimensión que punteó más bajo fue la afectiva y sexual $(50 \%)$.

La formación en el prenoviciado está compuesta por los cuatro pilares de la Orden, es decir, la vida comunitaria, la vida espiritual, la vida apostólica y la vida académica e intelectual. Cada pilar se constituye en una herramienta que nutre el ser y el quehacer como futuro religioso, razón por la cual lo principal en los procesos y en cada uno de los contenidos es la formación humana y cristiana, antes que dominicana. Es claro que los jóvenes que ingresan a este proceso de formación en el prenoviciado son laicos, no tienen ningún compromiso canónico, es decir, votos o promesas, que son necesarios para optar por la vida religiosa.

Basta ver el resultado de lo que significó para cada uno de ellos la formación integral, en donde se desarrolló, principalmente, la vida intelectual, moral, física y espiritual; el promedio fue del $94 \%$ en cada dimensión. 


\section{La organización o secuenciación}

El prenoviciado tiene una duración de un año, según lo establecido por la Provincia de Colombia y se realiza en la ciudad de Tunja (Boyacá), desde hace quince años. Actualmente está distribuido en cuatro trimestres, que van de febrero a enero del siguiente año. Cada trimestre hace énfasis en un pilar de la Orden y guarda una secuencia, a saber: vida comunitaria, vida académica, vida apostólica y vida espiritual. Una de las razones es el camino procesual que va desarrollando cada formando, sin descuidar los demás énfasis, ya que para un dominico debe existir un adecuado equilibrio entre los pilares, de lo contrario, sería ajeno a la auténtica espiritualidad de la Orden.

Así las cosas, el 97\% de los formandos consideró que hay una adecuada organización, coherencia y secuencia de las etapas de formación en el prenoviciado. Uno de los formandos afirmó que

Todos los aspectos se van desarrollando conjuntamente y de manera integral a su ritmo. Claro que si en el transcurso de ese año se vivieron por etapas cada una de ellas, sin olvidarlas en la etapa siguiente siempre estaban integradas, solo que se hacía más énfasis en la etapa establecida.

En una de las afirmaciones se planteó que:

Con la experiencia que tuve se llevó ese eje planteado y realmente fue coherente en su desarrollo y consecuente al final en cuanto a la vida espiritual, ya que nos preparó, en última instancia, al noviciado, y como bien sabemos en el noviciado se vive a profundidad el encuentro personal con uno mismo y con Jesucristo.

De igual manera, el 90\% de los encuestados respondió que es suficiente un trimestre para cada énfasis o pilar que se trabaja durante el año. Algunas de las razones son:

Pienso que la convivencia durante tres meses es suficiente para saber cómo se desenvuelve la persona, cuáles son sus actitudes, sus métodos, relaciones, espiritualidad, etc., el prenoviciado lo considero como un ciclo en el cual conoces de manera general todos los aspectos de la Orden, uno de ellos son sus pilares. 
A la pregunta de si existe una articulación en los procesos llevados a cabo entre el prenoviciado y el noviciado, el $47 \%$ cree que sí y el $20 \%$, no.

Algunas de las razones expuestas son:

- No son articulados, pues se llevan de forma muy independiente, $\mathrm{y}$ no se hacen integraciones entre las dos etapas, de manera que los prenovicios muy pocas veces saben el acontecer del noviciado hasta que vienen a él.

- Porque es una secuencia organizada, la formación del prenoviciado constituye las bases para desarrollar un buen noviciado, lo que implica que es una continuidad y no son procesos sueltos. Porque toda la formación del prenoviciado se proyecta hacia la construcción de valores humanos y cristianos enfocados en la vida consagrada.

\section{La metodología}

Vistas las anteriores partes del currículo, la relación de los formandos y los formadores tanto en el prenoviciado como en el noviciado y el estudiantado acontece en un ambiente familiar debido a la convivencia las veinticuatro horas del día.

Fundamentalmente debe existir una relación dialogal entre cada formando y los formadores, entre su nueva comunidad de hermanos y cada una de las herramientas que se brindan, a partir de la confianza recíproca.

Frente al acompañamiento y discernimiento vocacional, la encuesta registró que

Los diálogos tanto con el maestro como con los psicólogos juegan un papel importante en este aspecto. En estos se trataron temas concernientes a las dificultades y debilidades a trabajar. Lo cual ayudó a la madurez vocacional. Y aportó a la decisión libre y sin coacción alguna de ingresar al noviciado. 
En tal sentido, el $100 \%$ de los encuestados considera que vivió una formación personalizada, comunitaria e igualmente fue importante la comunidad formadora, entendida no solo como la participación del maestro de prenovicios y su socio, sino que tuvo en cuenta a los demás frailes del Convento con los que establecieron adecuadas relaciones de diálogo y acompañamiento tanto espiritual como académico.

El 97\% cree que los pilares o elementos del carisma de la Orden deben hacer parte del proceso y de las etapas o ciclos de la formación en el prenoviciado.

El $100 \%$ opina que el proceso plasmado en el proyecto personal de vida es importante en su formación.

El 100\% sostiene que se ofrecieron alternativas de apoyo personalizado en las dimensiones de la vida humana que lo requerían.

El $97 \%$ piensa que se facilitaron los medios y las experiencias necesarias para fortalecer su vínculo con el Señor Jesús, la vivencia del Evangelio, las prácticas sacramentales, en especial la Eucaristía y la Reconciliación; dando fundamento a la vivencia espiritual propia de la Orden.

El 100\% afirma que se profundizó en el reconocimiento y orientación de las aptitudes y capacidades de cada uno para la vida de estudio, el cultivo de los mismos, la indagación asidua del saber, y suplir las deficiencias académicas y culturales diagnosticadas en el proceso de acompañamiento vocacional.

El 100\% cree que se le acompañó en la formación de la conciencia autónoma, la autocrítica reflexiva y ponderada, el desempeño de responsabilidades delegadas, el ejercicio del liderazgo, la subsidiariedad y el rendimiento de cuentas, en procura del bien común.

En síntesis, los formandos consideran como valioso el proceso llevado a cabo en el prenoviciado, de manera especial se destacan la vida comunitaria y la vida académica con el $95 \%$, seguidos de la vida de oración y la vida apostólica, así como el acompañamiento de los formadores con un $94 \%$. Habrá que propender por la interacción y el conocimiento de la ciudad de Tunja, quizá con la participación en las distintas actividades que hay en la ciudad, puesto que solamente el $1 \%$ considera valioso este tema. Vale la pena resaltar 
que a través de las cátedras de historia de las civilizaciones, música y cultura física se participa de la vida cultural, histórica y deportiva de la ciudad.

A la pregunta “¿Qué considera como lo más difícil de la experiencia en el prenoviciado?”, se registran tres respuestas:

- "La adaptación al clima, al ritmo académico, al horario y al deporte”.

- "El adaptarme a una vida lejos de mi familia".

- "Empezar a adecuarse a un estilo de vida comunitario, muy marcado por los horarios".

Por otro lado, en relación con la pregunta sobre qué considera que fue lo más agradable de la experiencia en el prenoviciado, se destacan las siguientes respuestas:

- "El fortalecimiento de una vida fraterna con la comunidad [de] formadores. El poder de nivelar y acrecentar mi intelecto en algunas asignaturas. El crecimiento humano y cristiano".

- "La madurez que se le ha venido dando a cada uno de los campos dentro de la formación integral”.

- "La experiencia de fraternidad, el conocer nueva gente, la experiencia de oración y el encuentro de familias".

\section{Los recursos didácticos}

La didáctica entendida como el "arte de enseñar”, según la Real Academia de la Lengua ${ }^{10}$, hace referencia a los elementos con los cuales se acompañan, guían y orientan los procesos de enseñanza y aprendizaje a través del desarrollo de actividades en todas las dimensiones que se plantean para la formación integral.

Es cierto que se considera un arte, pero estaría muy limitada una comprensión del sentido y la esencia de la didáctica, por ello, al ampliar

10 Real Academia de la Lengua. Consultado en <http://lema.rae.es/ drae/?val=didactica . 
este concepto, se puede decir, siguiendo a Elvia Villalobos, que la didáctica "es el conjunto de etapas que se suceden para que se genere el binomio entre la enseñanza y el aprendizaje, con la interrelación de todos los elementos que intervienen en ellas" ${ }^{11}$.

La didáctica, como uno de los elementos del currículo, ayuda a ordenar, orientar, relacionar y crear, de manera dialogal, ciertos ambientes que propician y enriquecen los procesos de enseñanza y aprendizaje que, en palabras de Villalobos, presenta lo valioso para llevar a cabo procesos de enseñanza (actos de enseñar) y de aprendizaje (actos de aprender), para la formación (actos de formar, de educar) de la persona ${ }^{12}$.

Dentro de los considerados agentes y ámbitos de la formación en el prenoviciado, sobresalen, en primer lugar, el llamado que Dios hace al seguimiento de Jesús, una experiencia iluminada por el Espíritu Santo, razón por la cual se comprende como una de las principales motivaciones espirituales que permiten el desarrollo y la recta intención, vivida a través de las motivaciones humanas del formando. Así las cosas, es el Espíritu Santo quien enseña, llama y guía, son convicciones que, debido a la fe, una persona cristiana considera fundamentales a la hora de optar o tomar una decisión.

De igual manera, por ser una Orden Mariana, propagadora del Santo Rosario, la Virgen María es considerada como modelo de consagración a Dios y de servicio a la humanidad.

En Domingo de Guzmán, acontece y nace la Orden de Predicadores con una espiritualidad cuyo fin principal es la salvación de la humanidad, en este caso, a través de la predicación de la Buena Nueva; siendo este el camino hacia la identificación con el carisma propio de los dominicos, con lo cual se muestra, a través de la formación en el prenoviciado, un carisma que está armónicamente equilibrado en cada uno de los pilares: vida común, vida espiritual, vida intelectual y vida apostólica. En últimas, viviendo y alimentando cada día el lema de

11 Villalobos Pérez-Cortés, Elvia M. Didáctica integrativa y el proceso de aprendizaje. México, Trillas, 2011, pp. 45-46.

12 Ibíd. 
"contemplar y dar a los demás el fruto de lo contemplado", como lo afirma el Aquinate.

Por su estructura y su forma de gobierno, la Orden de Predicadores posee un sistema democrático, el cual está constituido por los frailes que pertenecen a ella, elegidos para participar en un Consejo que se organiza a nivel de Provincia, Convento o Casa. En este sentido, una casa de formación, como es el Convento de Santo Domingo de Tunja, se constituye en una comunidad formadora que acompaña y anima los procesos vocacionales de los prenovicios.

En tal sentido, el 100\% de los encuestados considera a la Comunidad Formadora, es decir, los formadores (maestro y socio), el Consejo de Formación Local, conformado por el prior del Convento, el promotor vocacional, el socio del maestro de prenovicios y el maestro de prenovicios quien lo preside, como una comunidad que acompaña y anima en el proceso de discernimiento vocacional.

El $97 \%$ de los encuestados valora positivamente el acompañamiento y discernimiento con el que se ven confrontados y animados por parte de los formadores, es decir, de los responsables de la casa de formación (maestro de prenovicios y su socio).

El acompañamiento psicológico en el prenoviciado es una oportunidad que el $93 \%$ los encuestados valoró, como una oportunidad para conocerse más y para que, en la absoluta confianza con los profesionales que han sido contratados por la Provincia, puedan avanzar en sus calidades humanas tanto a nivel personal y familiar como comunitario.

Es significativo el acompañamiento por parte del Departamento de Bienestar Universitario de la UsTA-Tunja, el cual cuenta con diferentes espacios para la programación de actividades lúdicas, culturales, de prevención en salud, entre otros.

Como lo señala el Capítulo Provincial de Bucaramanga ${ }^{13}$, solicitó al maestro de prenovicios que "siga promoviendo con el mismo entusiasmo [...], los encuentros con las familias de los prenovicios”. A la pregunta sobre los encuentros con las familias y el apoyo, los formandos

13 Provincia de San Luis Bertrán de Colombia (PSLBC), Actas del Capítulo Provincial. Bucaramanga, PSLBC, 2010, n. ${ }^{\circ} 144$. 
encuestados consideran que esto les permitió fortalecer la construcción de su proyecto de vida $(27 \%)$, construir y creer en su opción vocacional $(27 \%)$ y restablecer las relaciones familiares $(7 \%)$.

En cuanto a los recursos para el acompañamiento y discernimiento vocacional se encuentran los siguientes aspectos:

El cronograma de actividades: herramienta de planeación que estructura de manera sistemática cada uno de los momentos y objetivos que están directamente relacionados con la etapa de formación en el prenoviciado.

Los horarios del prenoviciado: con los cuales se establece una disciplina, una organización que tiene que ver con la vida cotidiana de una comunidad como, por ejemplo, el deporte, la oración, el estudio, la alimentación, el descanso, los encuentros fraternos y el aseo de la casa de formación.

El hábito de estudio, la lectura y la reflexión: a través de los tiempos y espacios destinados para el desarrollo de las actividades en el aula, la sala de sistemas y la biblioteca, entre otros, se orienta y guía en la lectura y la búsqueda de textos acordes con el pénsum académico y los énfasis que trimestralmente se desarrollan en el año de formación.

Las salidas comunitarias: son espacios que permiten la interacción y el conocimiento de los formandos y de los formadores, que se llevan a cabo fuera del lugar físico en el que se encuentra la casa de formación.

La planta física del prenoviciado: un espacio construido con una arquitectura moderna. Consta de espacios para el estudio, como son el aula de clases, auditorio, sala de sistemas y biblioteca, capilla, cafetería y salas de televisión y juegos, espacios deportivos y las habitaciones dentro de los claustros con que cuenta el edificio. De igual manera, se tiene acceso al Campus de la Universidad Santo Tomás, lugar que cuenta con adecuados equipos, canchas deportivas y asistencia médica.

El $90 \%$ de los formandos cree que el principal responsable de la formación es él mismo. Algunas de las razones son el haber recibido una formación de la conciencia autónoma, la autocrítica reflexiva y ponderada, el desempeño de responsabilidades delegadas, el ejercicio del liderazgo, la subsidiariedad y el rendimiento de cuentas en procura del bien común. El 100\% de los encuestados así lo registró. 
Finalmente, el 100\% de los formandos cree que la construcción y el desarrollo del proyecto de vida, como una estrategia para el acompañamiento vocacional, es una herramienta en donde se plasma su pasado, su presente y su futuro, a través de los espacios, tiempos, etapas y seguimiento constante a través de los diálogos con los formadores y con los nuevos hermanos de comunidad.

\section{La evaluación}

La evaluación como componente integral del currículo se establece en nuestro contexto de formación en el prenoviciado, dentro del paradigma antropológico, cualitativo y cuantitativo. Su combinación obedece al tipo de procesos y a la formación integral que se desarrolla con cada uno de los formandos, que tiene como fin acompañar y orientar en el discernimiento vocacional de los jóvenes que ingresan, sumado a las exigencias del sistema educativo que el Ministerio de Educación Nacional plantea en el caso de las instituciones de Educación Superior.

Se trata no solo de evaluar conocimientos, habilidades, destrezas y actitudes, sino de establecer una coherencia entre las prácticas pedagógicas y la teoría, que permitan tanto en el formando como en la comunidad formadora la capacidad para cambiar, de manera argumentativa y crítica, sus estructuras de pensamiento.

En nuestro caso, la evaluación se entiende como una acción de mejoramiento continuo, por procesos y en comunión con el modelo pedagógico dominicano-tomista, en el que se desarrollan de manera integral y equilibrada los cuatro ejes transversales de la formación: humana, religiosa, intelectual y pastoral.

Dentro de los elementos a tener en cuenta en la evaluación, aparecen algunos lineamientos que nos permiten orientar dicho proceso de mejoramiento, como son: ¿qué evaluar?, ¿cuándo evaluar? y ¿cómo evaluar? Se trata de una evaluación sistemática estructurada a partir de cuatro momentos ${ }^{14}:$ ) evaluación del contexto (valoración del formando a nivel familiar, económico, político, académico, religioso,

14 Stufflebeam, Daniel; Shinkfield, Anthony. Evaluación sistemática. Guía teórica y práctica. España, Paidós, 1995. 
afectivo, etc.); 2) evaluación de entrada o inicial (el presente del formando, sus expectativas, ilusiones, y proyecto de vida); 3) evaluación del proceso (es decir, el presente y cada una de las etapas que está viviendo el formando en el prenoviciado); y 4) evaluación final (resultado del proceso de formación en el prenoviciado para optar o no, libre y voluntariamente, por el noviciado).

Todos los procesos y las etapas de formación son evaluados cada trimestre. Allí se establecen los siguientes criterios para llevar a cabo cada objetivo de la etapa a partir de los logros, debilidades y sugerencias, según se muestra a continuación:

Evaluación de las comisiones: cada semestre se nombran los integrantes para la comisión de vida comunitaria, vida apostólica, vida intelectual, vida espiritual.

Evaluación de los oficios: en el prenoviciado se han establecido algunas responsabilidades que tienen que ver con la dinámica del grupo y de las actividades cotidianas que ocurren. En este caso, cada formando tiene una responsabilidad, un oficio, que es evaluado por el grupo bajo los mismos criterios de logros, debilidades y sugerencias.

Evaluación de los apostolados: al iniciar cada semestre los formadores asignan los grupos que participarán de manera activa y creativa en los lugares que la Iglesia particular de Tunja posee y que permiten el comienzo y la interacción con una manera de sensibilizarse, con el objetivo de despertar el celo apostólico, el trabajo con los más necesitados, los pobres, los enfermos y los grupos que desean participar de las catequesis presacramentales.

Evaluación de los docentes y formadores: a partir de una rejilla, se aplica cada semestre este instrumento, el cual aborda los siguientes elementos: el programa y su desarrollo, trabajo presencial y tutorial, actitudes, entre otros. Para los formandos, resulta importante este ejercicio en cuanto pueden intervenir en el mejoramiento de las prácticas pedagógicas de los docentes.

Desarrollo del proyecto de vida: desde el momento en que ingresa el prenovicio, se dan las herramientas necesarias para comenzar a articular y escribir sobre su proyecto de vida: pasado, presente y futuro, a partir del contexto familiar, social, académico, político, religioso, económico y afectivo. 
El 100\% de los formandos considera que la evaluación de los procesos y las etapas en el prenoviciado son importantes para mejorar y superar algunas situaciones tanto favorables como desfavorables.

El $97 \%$ cree que la evaluación bajo los criterios de logros, debilidades y sugerencias es la más adecuada si se tiene en cuenta que se está construyendo comunidad.

Finalmente, el $100 \%$ del grupo encuestado cree que es importante evaluar los oficios, los apostolados y las comisiones que integran, dinamizan y animan la vida cotidiana del prenoviciado.

La evaluación personal de cada formando se realiza a partir de los avances, logros y conocimiento tanto personal como comunitario por parte de los formadores y de los integrantes del grupo de prenovicios, a partir de su desempeño, progreso, dificultades, entre otros, que se ven registrados en el proyecto personal de vida, el cual se revisa con frecuencia junto con los formadores.

La encuesta finalizó con el tema de la formación humana integral. $\mathrm{Al}$ respecto, se planteó la afirmación de Santo Tomás de Aquino sobre la educación: "Conducción y promoción de la prole al estado perfecto del hombre que, en cuanto hombre, es el estado de virtud", es decir, la educación tiene unas intenciones en la formación del ser humano, posibilitando el desarrollo holístico de sus dimensiones. Educar en las virtudes es aunar esfuerzos para que la persona complemente sus capacidades en la medida que no es perfecto, se está haciendo, nunca acabado.

El $100 \%$ de los encuestados cree en esta afirmación, la vive y se encuentra en la búsqueda de ser personas virtuosas.

En la parte final de la encuesta se dejó una pregunta abierta a los encuestados: “¿Qué aportes nos puede brindar a partir de sus experiencias para la construcción de un Plan de Formación para el prenoviciado?”. Al respecto, resaltamos algunas:

- "Que en el desarrollo de este plan intervengan especialmente todos los formadores de las diferentes etapas de formación”.

- "Que se trabaje profundamente en la afectividad e identidad sexual". 
- "Es importante enfatizar en la libertad del manejo del ocio, para esto hay que brindar espacios en los que se practique la responsabilidad, libertad y autonomía. Continuar desarrollando actividades que permitan la unidad del grupo y el fortalecimiento de lazos de fraternidad entre los prenovicios”.

- "Propiciar un espacio o núcleo flexible que permita potenciar y aplicar los conocimientos que cada persona trae, especialmente para los profesionales".

- "Que se continúen realizando actividades que promuevan el crecimiento humano y vocacional, que permitan afianzar nuestra vida cristiana, de oración, académica, apostólica y comunitaria”.

Con seguridad, estos elementos enriquecen la propuesta para un plan de formación que continúe aportando en los procesos de acompañamiento y discernimiento vocacional de los futuros frailes.

En síntesis, los resultados nos permiten alimentar una propuesta curricular para la formación de los prenovicios dominicos en Colombia, a partir del ejercicio de triangulación entre los lineamientos que plantea el Magisterio de la Iglesia y la Orden de Predicadores para la formación integral, los modelos pedagógicos y los elementos curriculares y, finalmente, el análisis de los resultados de la investigación aplicados a través de cuestionarios y resúmenes analíticos, generando dicha propuesta. 
Ilustración 9. Triangulación del trabajo de investigación

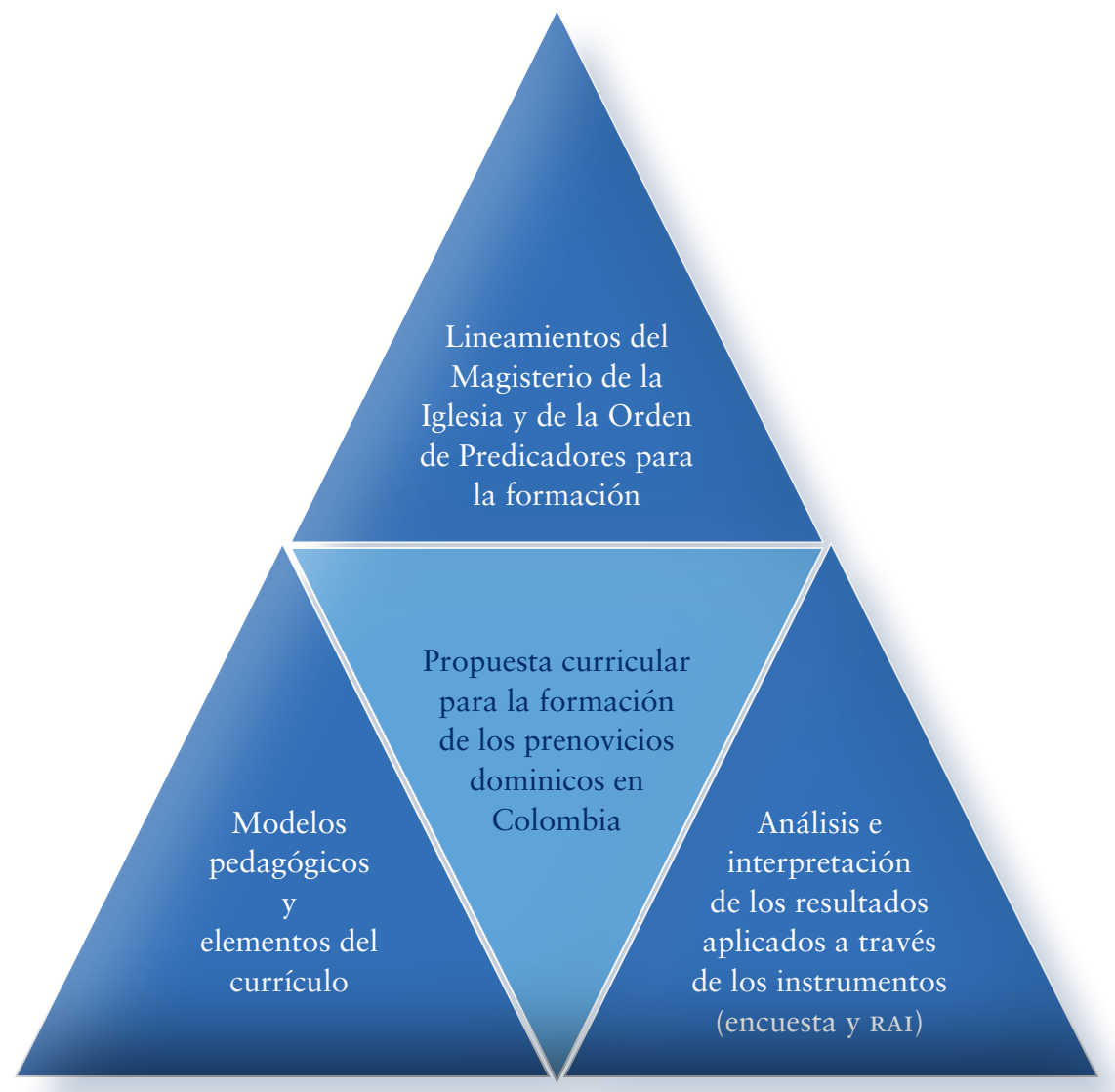

Fuente: elaboración del autor. 